\title{
Combatting Rose Rosette Disease with Volunteer Involvement in the Monitoring Effort
}

\author{
Kevin Ong ${ }^{1}$ and Madalyn Shires \\ The Texas Plant Disease Diagnostic Laboratory, Texas A\&M AgriLife Extension Service, Texas \\ A\&M University, College Station, TX 77843 \\ Holly Jarvis Whitaker \\ Texas A\&M AgriLife Communications, College Station, TX 77843
}

\author{
Jennifer Olson \\ Department of Entomology and Plant Pathology, Oklahoma State University, Stillwater, OK 74078 \\ Joseph LaForest \\ University of Georgia Extension, Center for Invasive Species and Ecosystem Health, Tifton, GA 31793 \\ David H. Byrne \\ Department of Horticultural Sciences, Texas A\&M University, College Station, TX 77843
}

Additional index words. rose rosette disease, Rosa, citizen scientist, disease distribution

\begin{abstract}
Rose rosette disease (RRD) was first reported on the North American continent in the early 1940s. In 2011, the causal agent of this disease was identified and described - the Rose rosette virus (RRV). In the last 10 years, RRD has gained widespread notoriety because of disease symptoms appearing on many roses which are used frequently in landscape plantings, both commercial and residential. Much of the prior scientific work on this disease was carried out on the multiflora rose. Currently, the disease issues are on cultivated roses within which no cultivar has been confirmed to be resistant. There is an information gap in our knowledge of the pathogen, vector, and the disease on cultivated roses. Our goals for this project are to seek and identify potential disease tolerance or resistance in roses and increasing public awareness and knowledge of RRD with the purpose of reducing the disease spread with best management practices. Outreach and volunteer recruitment are key activities used to provide scientifically sound information, to establish the current disease range and to actively gather observational reports of RRD to identify resistant rose sources. Elements of these activities include educational meetings, factsheets, posters, and workshops where RRD symptoms recognition is emphasized. A web-based reporting tool was developed to capture observations from volunteers while continually keeping them engaged. It is hoped that through outreach and the collective monitoring effort, researchers will have access to information that contributes to a better understanding of RRD and will find disease-resistant roses that could be used in breeding programs for the continued enjoyment of roses.
\end{abstract}

Rose rosette disease was described in the early 1940s (Conners, 1941; Thomas and Scott, 1953). Since then, much of the work that was carried out to understand this disease was focused on multiflora roses until the 1990s. In recent years, RRD became a growing problem on cultivated roses and continues to be a problematic plant disease issue in many parts of the United States of America. It was quickly realized that there is a large information gap because of the lack of knowledge of RRD in cultivated roses. In 2014, the project entitled "Combating Rose Rosette Disease: Short-term and Long-term Approaches" was funded by a National Institute of Food and Agriculture

Received for publication 2 Oct. 2017. Accepted for publication 1 Mar. 2018.

This paper is part of the workshop entitled, The Challenges of Rose Rosette Disease (RRD): An Update of the Combating RRD SCRI Project, presented on 9 Aug. 2016, during the ASHS Annual Conference, Atlanta, GA.

This work was funded by the USDA's National Institute of Food and Agriculture (NIFA) Specialty Crop Research Initiative project "Combating Rose Rosette Disease: Short Term and Long Term Approaches" (2014-51181-22644/SCRI).

${ }^{1}$ Corresponding author. E-mail: kevo@tamu.edu.
Specialty Crop Research Initiative grant. A component of this project is to identify potential sources of RRD resistance in cultivated roses. The strategy used to accomplish this goal is to engage the public to assist in the monitoring effort for RRD. Because of the widespread nature of this disease, we believe that recruitment and engagement of the general public in a monitoring effort will enhance our ability to assess and establish the current range and monitor the progression of RRD in the United States. Utilization of the general public to assist in monitoring effort is not novel. The use of volunteers in scientific efforts, known as citizen science, has benefitted and advanced science in areas of conservation science, natural resource management, ecological research, biodiversity research, and others (Flannery, 2016; McKinley et al., 2016; Tulloch et al., 2013). However, numerous challenges exist when attempting to engage the general public in any research program. This article will explore the logic and development of approaches of using citizen science in the Combating Rose Rosette Disease project.

For a successful public engagement effort, clear objectives must be defined for the monitoring effort. What is the information that we would like to gather? With a large number of cultivated roses available, we would like to accelerate our progress to identify cultivars susceptible to RRD so that we can determine if any natural resistance, tolerance, or both exist among currently available rose cultivars. All cultivated (hybrid) roses are believed to be susceptible, but some native roses have been reported to show no symptoms of this disease (Epstein and Hill, 1999). If tolerance, resistance, or both are found in currently available varieties, it would provide an advanced platform from which to develop a breeding strategy to create resistant roses with desirable characteristics.

\section{MATERIALS AND METHODS}

Volunteer recruitment and retention. Although the primary goal of the monitoring effort is to seek and identify potential tolerance/resistant germplasm, utilization of citizen scientists (public volunteers) is a strategy used to accelerate this effort. A knowledgeable and enthusiastic citizen scientist is needed for quality data. As such, the secondary goal of this project is to develop more RRD awareness and to equip interested individuals with the knowledge to identify RRD to assist in our efforts to seek and 
identify potential RRD-resistant roses. Our targeted demographic includes people who are interested in gardening and with a particular interest in roses. Targeted groups include, but are not limited to, Master Gardeners, rosarians, and garden club members.

A primary avenue for volunteer recruitment is awareness meetings. These face-toface or group educational events allow for the delivery of basic information about RRD. This would include basic pathogen biology and epidemiology, and symptom recognition. These meetings also allow for the exposure of the volunteers to the reporting tool (digital data entry system) to encourage familiarity and reduce potential fear and anxiety of the technology. In addition to RRD awareness, participants are taught about other rose disease and pest issues. This value-added information contributes to developing participant's knowledge about rose health.

To maintain continued excitement and enthusiasm in volunteers, we plan to actively engage the volunteers with continuous education opportunities. Educational opportunities include e-learning opportunities, webminars, face-to-face trainings, and the availability of information via fact sheets and research progress reports via social media.

A rose rosette e-learning module, which provides information about symptomatology and best management practices, has been developed and was deployed with the launch of the roserosette.org website. Learning outcomes for the e-learning module include articulating what RRD is and how it spreads, listing RRD symptoms, differentiating between RRD and look-alike problems, creating an action plan if RRD is suspected, prioritizing actions taken to prevent the spread of RRD, and recognizing the procedure for reporting suspicious symptoms via the web-based reporting tool. Evaluation of learning outcomes is achieved through Kirkpatrick and Kirkpatrick (2006) first and second levels of evaluation. Level 1 or feedback on customer satisfaction evaluation will assess the final learning outcome of prioritizing action, whereas Level 2 or assessment of knowledge gain will yield data on the other learning outcomes. All evaluation data will be collected using a multiple-choice quiz given to learners at the end of the module. This module can be accessed at http://bit.ly/ RRVlearn.

Factsheets on RRD and some other rose diseases are currently available and distributed. Additional factsheets are being developed, whereas others are being updated to reflect current knowledge and management methods. These documents provide a reminder to volunteers that the health of the rose plant can be affected by other pests and pathogens, and all damage is not the result of RRD alone. These factsheets can be accessed from the http://roserosette.org website.

Tool for information gathering. A robust, and yet, user-friendly tool must be developed to encourage involvement by public volunteers. Information that is targeted in this project are cultivar identification and location. Cultivars that are identified and verified as RRD susceptible will help to narrow down the number of cultivars that would be included into future disease resistance trials, thus accelerating our ability to identify potential RRD resistance, tolerance, or both in roses. Locations of verified RRD diseased plants help to understand the current range and distribution of this disease.

This effort is being accomplished through a web-based tool developed by the University of Georgia's Center for Invasive Species and Ecosystem Health. It expands on the existing UGA platform to take advantage of developed tools, such as reporting, verification of data, and mapping with additional functionality, for routing information and samples to diagnostic laboratories. The data entry system is optimized for volunteers to use on any web browser on their digital device, such as smartphones, to allow for reporting and submitting photos. It allows for quick and easy reporting by volunteers to encourage participation. This tool has the potential to capture higher quality data such as images of suspect diseased roses, reduce potential data entry errors, and identify locations accurately. The reporting tool can be accessed at http://roserosette.org/reporting/.

Verification and ensuring quality data collection. Educating the volunteer is the first step in ensuring that quality data would be submitted. The reporting tool was developed with the understanding that reported observations ought to be vetted. A logical information vetting model was designed to address the concern of reliable observation data (Fig. 1). Briefly, initial evaluation of the submitted observation data will be made by diagnosticians, researchers, and other trained screeners, collectively known as "verifiers." Verifiers may make determinations based on the symptoms that are seen on the submitted information and photos. If submitted photos exhibit symptoms that are highly suspicious of RRD but the verifier is unable to conclusively determine that RRD is the cause, verifiers may request that a physical sample be submitted for confirmatory diagnosis. At this point, this entry may be classified as "plausible" or "credible." Although we recognize that this diagnostic approach is subjective, the use of verifiers helps to reduce misidentification, confirm obvious RRD cases, or both. In the event that RRD is reported on a previously unreported cultivar, verifiers can request samples of the suspect plant to be sent to diagnostic laboratories for confirmation. The reporting system will generate a response to the submitter/reporter with a simple protocol for collecting a sample, a packing slip to accompany the sample, and a shipping label to direct the sample to the appropriate laboratory based on their location. On receipt of the sample, the laboratory can use the packing slip to access and view the existing submission information. Once the confirmation testing is completed and the result is entered, notifications are sent to verifiers and reporters to provide outcome information. Conclusions from verifiers and confirmation results are used by the reporting tool to update distribution and incidence maps.

\section{RESULTS AND OUTCOMES}

Outreach activities such as awareness educational meetings which include RRD information have been delivered by extension and research personnel since the mid2000s. Often RRD information was a minor component in the educational programs. In recent years, interest in RRD has increased tremendously as incidences of this disease on cultivated roses have become more apparent and highly visible. Since 2016, we estimate that at least 50 educational programs specifically focused on RRD were delivered in Texas and Oklahoma by extension specialists and county agents.

The Rose Rosette website (http://roserosette.org) was launched in early 2017 to function as a single-site information clearinghouse

\section{Monitoring App}

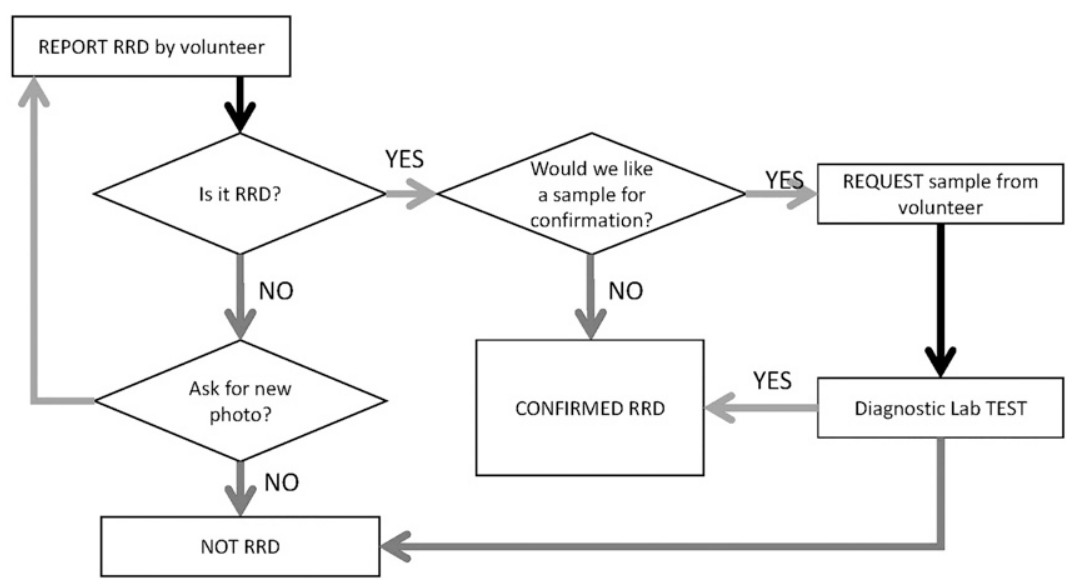

Fig. 1. Monitoring (reporting) app on the roserosette.org website vetting model. This is the proposed flow of how reports from the public will be managed to capture new observations of rose rosette disease (RRD). 
for RRD information and outreach from the Specialty Crop Research Initiative Combating Rose Rosette project. This is a continuous effort as additional information, links to collaborators, and other related RRD information are added. This site will serve as a portal to state-specific information and general educational materials. It is also used to collect and consolidate current observations and reported RRD incidences. To date (15 Mar. 2018), we have over 1205 records, which includes historical data from the literature that is manually inputted, observations through personal communication with colleagues, and reports received (and verified) through the website RRD reporting tool. The RRD reporting tool was launched in mid-2017, where a major marketing push for the reporting site commenced in the fourth quarter of 2017.

A major challenge in RRD diagnosis is that many RRD symptoms can be mimicked by other causes, such as herbicide damage, nutrient deficiencies, pest damage, other pathogens, or all of these. Before 2011, the diagnosis of RRD was made primarily on the observation of symptoms alone. In 2011, a previously undescribed pathogen, RRV, was identified as the causal agent of RRD (Laney et al., 2011). This report provided, for the first time, an approach using a polymerase chain reaction (PCR) technique to detect the presence of RRV. One aim of this monitoring project is to provide confirmation of infection when identifying susceptible cultivars. This will provide the ability to differentiate between observational and PCR-confirmed reports, thus providing greater confidence in recognizing resistant cultivars.
At the time of this writing, analysis of all the records in the reporting database indicates 586 susceptible rose cultivars based on observed symptoms. One hundred and twentyeight of these were confirmed by PCR to be susceptible to RRD.

In the short period that the RRD reporting tool has been available, 64 reports have been submitted, resulting in 11 confirmed (by visual observation/photographs), 29 plausible or credible reports, and one PCR-confirmed report. It has also resulted in three negative reports based on visual observation, PCR, and expertise. This tool also captured reports of RRD from five previously unrecorded counties/states. It is anticipated that this tool will continue to assist in our ability to gather information that will help to inform and lead to rose cultivars that are resistant, tolerant, or both for breeding programs.

\section{CURRENT AND FUTURE EFFORTS}

There is a current effort to provide the best information on RRD and best management practices based on current scientific research and using the roserosette.org website as an information clearinghouse. There is also an active effort to promote the use of the reporting tool and the educational materials that are available in this website. There will be continued efforts to develop educational materials, such as e-learning modules and factsheets, on various topics related to rose health management. Recently, Star Roses and Plants helped to host a webminar on RRD targeting landscape professionals. Currently, several face-to-face training programs are planned to reach the general public this spring. Through these efforts, we hope to excite and increase participation of volunteers to assist in our efforts to seek and identify potential RRD-resistant roses.

\section{Literature Cited}

Conners, I.L. 1941. Twentieth annual report of the Canadian plant disease survey 1940, Vol. 20 Div. Bot. Plant Pathol., Domain Can. Dept. Agr. Sci. Serv., Ottawa, Canada.

Epstein, A.H. and J.H. Hill. 1999. Status of rose rosette disease as a biological control for multiflora rose. Plant Dis. 83:92-101.

Flannery, M.C. 2016. Citizen science helps botany flourish. Amer. J. Bot. 62(1):10-15.

Kirkpatrick, D.L. and J.D. Kirkpatrick. 2006. Evaluating training programs: The four levels, third edition. Berrett-Koehler Publishers, San Francisco, CA.

Laney, A.G., K.E. Keller, R.R. Martin, and I.E. Tzanetakis. 2011. A discovery 70 years in the making: Characterization of the Rose rosette virus. J. Gen. Virol. 92:1727-1732.

McKinley, D.C., A.J. Miller-Rushing, H.L. Ballard, R. Bonney, H. Brown, S.C. Cook-Patton, D.M. Evans, R.A. French, J.K. Parrish, T.B. Phillips, S.F. Ryan, L.A. Shanley, J.L. Shirk, K.F. Stepenuck, J.F. Weltzin, A. Wiggins, O.D. Boyle, R.D. Briggs, S.F. Chapin Iii, D.A. Hewitt, P.W Preuss, and M.A. Soukup. 2016. Citizen science can improve conservation science, natural resource management, and environmental protection. Biol. Conserv. 208:15-28.

Thomas, H.E. and C.E. Scott. 1953. Rosette of rose. Phytopathology 43:218-219.

Tulloch, A.I.T., H.P. Possingham, L.N. Joseph, J. Szabo, and T.G. Martin. 2013. Realising the full potential of citizen science monitoring programs. Biol. Conserv. 165:128-138. 